\title{
View from Europe: new dynamics*
}

Chair:

Georg Schultheiss, Chairman of EUSIDIC

Schultheiss continues the theme of the 1998 EUSIDIC Conference: The Dynamic of the Emerging Information Society in this moderated discussion on EU initiatives including the Transatlantic Business Dialogue

Speakers:

Arnoud de Kemp Director, Sales/Marketing and Corporate Development, Springer Verlag, HeidelPeter Shepherd $\quad$ Managing Director, Elsevier Science, Switzerland

\section{The European Scene}

European initiatives to develop an Information Society have resulted in the establishment of a Global Business Dialogue, explains Dr George Schultheiss, Chairman of EUSIDIC and Director of FIZ Karlsruhe. This Dialog has the lofty goal of harmonization, which is naturally complicated by the competitive business environment, cultural differences, and legal differences. The harmonization process aims to remove obstacles for access to and interactions in the digital and e-commerce arenas.

From the perspective of a global publisher based in Europe, Peter Shepherd, Managing Director of Elsevier Science Switzerland, sees three dynamics at work in the creation of an Information Society:

(1) The changing behavior and expectations of authors and users: Electronic communications media are enabling "invisible colleges" in science and research to operate more effectively and globally. In some cases, publishers are being cut out of the loop. Many electronic journals and preprint services are author initiated and publisher free. Now that publishers are disseminating their journals electronically, however, Shepherd doesn't anticipate a major shift of author behaviors away from established journals.

Scientists as users of information expect greater integration of information (scientific research is becoming more interdisciplinary), and they want information integrated at their desktop. They also want user-friendly navigation tools, access to archives, and more domain-specific content (graphics, etc.).

(2) The changing behavior of institutes: Academic universities are increasingly using consortia to negotiate license agreements. Institutes also want a greater choice in accessing the scientific literature. They want a mix of access and ownership, subscription and transactional business, and they want more value.

\footnotetext{
*In cooperation with the European Association of Information Services.
} 
In industry, there is an increasing pressure for a major company such as a pharmaceutical company to negotiate global licenses for sitewide access to information. There also is an increasing integration of internal and external information. And industry shows a lively concern regarding intellectual property rights.

The last ten years have witnessed the rise of small- to medium-size research companies with very different needs from the major academic institutions and industrial companies that also must be addressed.

(3) Changes in ownership of scientific information: There is an increasing concern about the consolidation of information in the hands of fewer publishers resulting from mergers and acquisitions. In the United States, paranoia is directed at the strength of European publishers dominating the scene. Europe mirrors this concern, fearing the domination of US societies.

Shepherd says that, through its strategy of significant acquisitions in recent years, Elsevier is better able to meet changing expectations by offering bigger, more integrated offerings - with more sophisticated delivery and navigational technologies.

Elsevier's push with electronic publishing is to integrate products and services into the daily work process of scientists. "Our ultimate objective is to help make the discovery process more efficient and productive", says Shepherd.

In terms of business directions, while the subscription model will continue to predominate in the future, Elsevier's Science Direct will change what is in the subscription package, including a variety of levels of access to the information. There will also be increasing transactional and advertising revenue from BioMedNet and ChemWeb. Shepherd recognizes that publishers may have to move to author funding to sustain some types of journals (those that can no longer survive on subscription income alone).

Arnoud de Kemp, Director of Marketing at Springer-Verlag, complements the previous perspective with the Dutch, German, and French publisher's view ... and a large dollop of humor. De Kemp makes the caveat that it is difficult to bring one unified European perspective to the table because there are many different cultures and languages operating under the "European" label. The European Commission is indeed very concerned that the English language predominates so largely on the Internet.

De Kemp reviews the changing dynamics of publishing, intermediaries, end users, institutional users, and technology.

In the past, publishing has been a quirky, time- and labor-intensive process, with little standardization and efficiency in the production mode. In the future, says de Kemp, the time to market - the speed of the publication process - is going to matter more and more. This demand is why we need enabling technologies and digital workflows in place. "We are only at the very beginning ..." reminds de Kemp.

Abstracting and indexing services as intermediaries are the ideal portal to all the information, says de Kemp. Publishers should work closely with A\&I services to provide fast answers to scientist's questions. Subscription services are also playing a bigger role in integrating and delivering information. Search engines such as Yahoo, Lycos, and Alta Vista reach no more than $30 \%$ of the sites; they are not nearly exhaustive enough.

The end users have reached critical mass. "But they are growing to realize that the new paradigms we are offering them do not represent a new paradise", notes de Kemp. There is a long way to go from text information to visual information, and "we need rules for information and publishing as a whole".

Libraries and consortia are becoming more important for licensing. There are regional and country licenses and even the development of The National Electronic Site License in Greece. Publishers should work more closely with libraries in linking to OPACs. 
"We know nothing about technologies. Everything we dream of today will be the laughing stock in five years", says de Kemp. So, for publishers, this is a very dangerous world, because there are no guarantees of what the next technology will bring. De Kemp does anticipate fairly swift acceptance of intelligent agents, open platforms among publishers, and electronic visas and passports (using a DPI digital personal identifier).

The only safety net for publishers - in trying to predict the future - is active market research. De Kemp advises all publishers to get involved in marketplace test/pilot projects. Springer-Verlag is involved in over 20 such RTD projects - projects in research, technology, and development. For example, Springer-Verlag, rather than negotiating full-term licenses on a permanent basis, Springer agrees to "test installations" that are monitored and adjusted over time. The result of specific Springer projects has been the first DOI implementation project called Online First, as well as a Bertelsmann-Springer health online service.

Other non-Springer RTD projects to watch are the Superjournal Project, the National Electronic Site License (NESLI) in Manchester, Germany's Global Info, and an interesting mathematics project in France. At the European Commission level, recent projects are ESPRIT, Fourth Framework, Liberation, Info 2000, and Information Engineering. Next week, the first call will come out from the Fifth Framework Program IST (Information Society Technologies).

Web look-ups: NBII: http://www.nbii.gov

GLOBAL BUSINESS DIALOG: http://www.gbd.org 